\title{
Poynting-Robertson effect and perihelion motion
}

\author{
J. Klačka ${ }^{1}$, M. Kocifaj ${ }^{2}$, P. Pástor ${ }^{1}$, and J. Petržala ${ }^{1}$ \\ 1 Department of Astronomy, Physics of the Earth, and Meteorology, Faculty of Mathematics, Physics and Informatics, \\ Comenius University, Mlynská dolina, 84248 Bratislava, Slovak Republic \\ e-mail: [klacka;pavol.pastor]@fmph.uniba.sk \\ 2 Astronomical Institute, Slovak Academy of Sciences, Dúbravská cesta 9, 84504 Bratislava, Slovak Republic \\ e-mail: astromir@savba.sk
}

Received 28 July 2006 / Accepted 17 October 2006

\section{ABSTRACT}

\begin{abstract}
The effect of stellar electromagnetic radiation on the motion of homogeneous spherical dust particles, the Poynting-Robertson $(\mathrm{P}-\mathrm{R})$ effect, is investigated. The effect already produces a shift of pericenter/perihelion in the accuracy to the first order in $\boldsymbol{v} / c$, where $v$ is velocity of the particle with respect to the source of radiation and $c$ is the speed of light. The advancement of pericenter occurs due to the fact that the P-R effect depends on radiation pressure efficiency factor $Q_{\mathrm{pr}}^{\prime}$, which is a function of optical properties of the particle. The particle orbiting a star moves in various distances from the star and the particle optical properties may vary with the distance, as they are functions of temperature.
\end{abstract}

Key words. scattering - solar system: general - interplanetary medium

\section{Introduction}

The effect of stellar electromagnetic radiation on the motion of homogeneous electrically neutral spherical dust particles is investigated. Although this effect is well-known as the PoyntingRobertson (P-R) effect (Robertson 1937; Klačka 1992, 1993, 2004), it was not recognized that it may also produce a shift of pericenter/perihelion in the accuracy to the first order in $v / c$, where $v$ is velocity of the particle with respect to the source of radiation and $c$ is the speed of light (Robertson 1937; Wyatt \& Whipple 1950; Lyttleton 1976). Klačka (2004) has shown that the shift of pericenter/perihelion exists if the central acceleration is given by the gravity of the central star. The general opinion is: if the central acceleration also contains a dominant part of the electromagnetic radiation pressure of the star, then no shift of pericenter exists, supposing that the equation of motion is considered within approximation to the first order in $\boldsymbol{v} / c$. In reality, the shift of pericenter/perihelion occurs. The reason is that the P-R effect depends on radiation pressure efficiency factor $Q_{\mathrm{pr}}^{\prime}$, which is a function of optical properties of the particle (Mishchenko 1991). The only exception is the idealized special case treated by Robertson, the perfectly absorbing sphere. The particle orbiting a star moves in various distances from the star and the particle's optical properties are functions of the distance, as they may depend on a temperature (corresponding physical equations determining the temperature of the particle can be found in Shulman 1987; see also Kocifaj et al. 2006; as for optical properties, see, e.g., Draine \& Lee 1984). This fact is not taken into account, in general (Lamy 1974; Kocifaj et al. 2006).

We consider the motion of spherical interplanetary dust grains around the Sun under the action of solar gravity and solar electromagnetic radiation. We do not assume that optical constants of the dust grains are independent of temperature, i.e. fixed in time, as it is usually done. Instead of that we consider the $\mathrm{P}-\mathrm{R}$ effect (to the first order in $v / c$ ) in its real physical form, when the efficiency factors for radiation pressure $Q_{\mathrm{pr}}^{\prime}$ are functions of heliocentric distance. We treat more realistic situations when the dielectric function of some dust constituents varies with temperature. As the P-R effect is dominant for the grains of radii between $1 \mu \mathrm{m}$ and $100 \mu \mathrm{m}$ (Leinert \& Grün 1990), we consider particles of radii within this interval. It turns out that the real P-R effect (with temperature dependence of optical properties) offers different behavior from the standard P-R effect (temperature independent optical properties) for small dust grains. We show this for small micron-sized dust spheres, while for compact dust grains with radii larger than $\approx 10 \mu \mathrm{m}$ the standard P-R effect can be used. Results of detailed numerical calculations are presented. Moreover, we try to understand/explain the obtained numerical results also in an analytical way. The analytical approach is in qualitative coincidence with the detailed numerical calculations, in general.

\section{Radiation and motion of a spherical particle}

Incident radiation acts by a force on a spherical particle. The dimensionless factor of the effectivity of radiation pressure is (see, e.g., Mie 1908, or, Sect. 4.5 in Bohren \& Huffman 1983)

$Q_{\mathrm{pr}}^{\prime}=Q_{\mathrm{ext}}^{\prime}-Q_{\mathrm{sca}}^{\prime}\langle\cos \theta\rangle$,

where $Q_{\text {ext }}^{\prime}$ and $Q_{\text {sca }}^{\prime}$ are dimensionless efficiency factors for extinction and scattering. Motion of the spherical particle in the reference frame of the source of radiation is given by the Poynting-Robertson effect. It reads, to the accuracy $v / c$,

$\frac{\mathrm{d} \boldsymbol{v}}{\mathrm{d} t}=Q_{\mathrm{pr}}^{\prime} \frac{S \pi R^{2}}{m_{\mathrm{p}} c}\left\{\left(1-\frac{\boldsymbol{v} \cdot \hat{\boldsymbol{S}}}{c}\right) \hat{\boldsymbol{S}}-\frac{\boldsymbol{v}}{c}\right\}$,

where $S$ is the flux density of radiation energy (energy flow through unit area perpendicular to the ray per unit time) as measured in the frame of reference of the radiation source, $v$ is the particle's velocity, $c$ is the speed of light. It is supposed that radiation is traveling in the direction and orientation given by the 
unit vector $\hat{\boldsymbol{S}}$. Particle mass $m_{\mathrm{p}}$ and the efficiency factor of radiation pressure $Q_{\mathrm{pr}}^{\prime}$ are particle properties given in the rest frame of the particle. The terms in brackets in Eq. (2) are generated by simultaneous action of the Doppler effect, the change of concentration of photons, and the aberration of light (see Sect. 3.4 in Klačka 2004). Another possible explanation of the PoyntingRobertson formula is following: the incident radiation generates force $\left(S \pi R^{2} / c\right)(1-\boldsymbol{v} \cdot \hat{\boldsymbol{S}} / c) \hat{\boldsymbol{S}}$ acting on the spherical particle, while the outgoing radiation acts on the particle with the force $\left(S \pi R^{2} / c\right)\left[\left(1-Q_{\mathrm{pr}}^{\prime}\right)(1-\boldsymbol{v} \cdot \hat{\boldsymbol{S}} / c) \hat{\boldsymbol{S}}+Q_{\mathrm{pr}}^{\prime} \boldsymbol{v} / c\right]$. However, the newest explanation that decomposition of the term $\boldsymbol{v} / c$ into radial $\boldsymbol{v} \cdot \hat{\boldsymbol{S}} / c$ and nonradial terms contributes to the existence of two radial terms in Eq. (2) and the presence of these two radial terms is "because of the combination of Doppler shift in absorbing radiation and Doppler shift in emission" (Quinn 2005, p. 195) does not correspond to the physical situation, as follows from the above presented physical explanation. (One may also consider special cases. A specular reflection on the mirror sphere yields $Q_{\mathrm{pr}}^{\prime}=2$ and incident and outgoing flows generate 1 and 3 radial terms $\boldsymbol{v} \cdot \hat{\boldsymbol{S}} / c$, respectively. In the case of a perfectly absorbing sphere $Q_{\mathrm{pr}}^{\prime}=1$ no term $1-\boldsymbol{v} \cdot \hat{\boldsymbol{S}} / c$ exists due to the outgoing radiation, as it would be required by Doppler effect: the existence of the term $1-\boldsymbol{v} \cdot \hat{\boldsymbol{S}} / c$ is a necessary, but not sufficient, condition for the presence of the Doppler effect.)

\section{Radiation and motion of a sphere around the Sun}

We are interested in a simultaneous motion of a spherical particle under the action of solar electromagnetic radiation and solar gravity. Equation of motion of the particle with respect to the Sun is

$$
\frac{\mathrm{d} \boldsymbol{v}}{\mathrm{d} t}=-\frac{G M_{\odot}}{|\boldsymbol{r}|^{3}} \boldsymbol{r}+\beta \frac{G M_{\odot}}{|\boldsymbol{r}|^{2}}\left\{\left(1-\frac{\boldsymbol{v} \cdot \hat{\boldsymbol{r}}}{c}\right) \hat{\boldsymbol{r}}-\frac{\boldsymbol{v}}{c}\right\},
$$

$\hat{\boldsymbol{r}} \equiv \boldsymbol{r} /|\boldsymbol{r}|$,

$\beta \equiv \frac{L_{\odot} \pi R^{2}}{4 \pi G M_{\odot} m_{\mathrm{p}}} \frac{\bar{Q}_{\mathrm{pr}}^{\prime}}{c}=7.6 \times 10^{-4} \bar{Q}_{\mathrm{pr}}^{\prime} \frac{\pi R^{2}\left[\mathrm{~m}^{2}\right]}{m_{\mathrm{p}}[\mathrm{kg}]}$,

where $G$ is the gravitational constant, $M_{\odot}$ is the mass of the Sun, $\boldsymbol{r}$ is the position vector of the particle with respect to the Sun, and $\bar{Q}_{\mathrm{pr}}^{\prime}$ is the spectrally averaged efficiency factor for radiation pressure. We have also taken into account that the flux density of radiation energy $S$, present in Eq. (35), is $S=L_{\odot} /\left(4 \pi|\boldsymbol{r}|^{2}\right)$, where $L_{\odot}$ is the solar luminosity.

\section{Numerical results}

Figure 1 depicts the dependence of the efficiency factor for radiation pressure $\bar{Q}_{\mathrm{pr}}^{\prime}$ on heliocentric distance for carbonaceous particles of radii $1 \mu \mathrm{m}$ and $2 \mu \mathrm{m}$. The changes of $\bar{Q}_{\mathrm{pr}}^{\prime}$ are important mainly for small heliocentric distances and $\bar{Q}_{\mathrm{pr}}^{\prime}$ is almost constant for distances greater than 5-10 AU. We want to stress that carbonaceous material was taken as an example for which refractive index is known for various temperatures (Jäger et al. 2003), while the dependence is not known for materials present in real astronomical dust grains (Kocifaj et al. 2006).

Table 1 presents several interesting values for carbonaceous particles. Besides radius $R$ and mass density $\rho$ of the particle, dimensionless efficiency factors for radiation pressure for the initial orbit of the particle $\left(\bar{Q}_{\mathrm{pr} \text { aph }}^{\prime}\right.$ at aphelion and $\bar{Q}_{\mathrm{pr} \text { per }}^{\prime}$ at perihelion) are also presented: a particle is ejected at the aphelion of

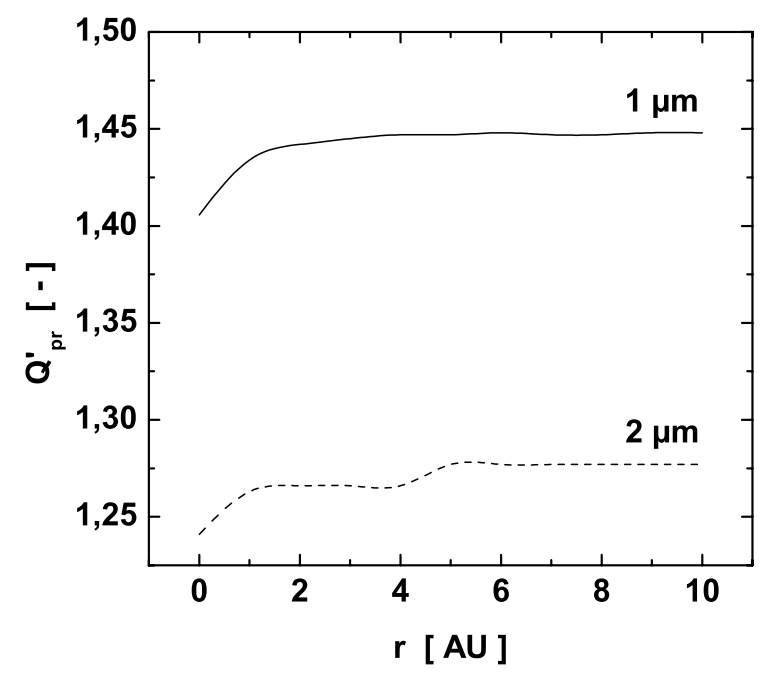

Fig. 1. Radiation pressure efficiency factor $\bar{Q}_{\mathrm{pr}}^{\prime}$ as a function of heliocentric distance for compact carbonaceous dust particles with radii $1 \mu \mathrm{m}$ and $2 \mu \mathrm{m}$.

Table 1. Characteristic parameters for carbonaceous spherical dust particle, including the shift of perihelion after 1000 years. $\bar{Q}_{\mathrm{pr}}^{\prime}$ values are given for initial orbit (0) and for the orbit after 1000 years (1).

\begin{tabular}{ccccc}
\hline \hline$R[\mu \mathrm{m}]$ & 1 & 1 & 2 & 2 \\
$\rho\left[\mathrm{g} / \mathrm{cm}^{3}\right]$ & 1 & 2 & 1 & 2 \\
$\bar{Q}_{\mathrm{pr} \text { aph }}^{\prime}(0)$ & 1.447 & 1.447 & 1.267 & 1.267 \\
$\bar{Q}_{\mathrm{pr} \mathrm{per}}^{\prime}(0)$ & 1.446 & 1.425 & 1.255 & 1.252 \\
$\Delta \omega\left[^{\circ}\right]$ & 249.8 & 511.4 & 304.2 & 132.2 \\
$\bar{Q}_{\mathrm{pr} \text { aph }}^{\prime}(1)$ & 1.447 & 1.441 & 1.266 & 1.266 \\
$\bar{Q}_{\mathrm{pr} \text { per }}^{\prime}(1)$ & 1.445 & 1.423 & 1.254 & 1.251 \\
\hline
\end{tabular}

a parent body characterized with orbital elements $a=2.2 \mathrm{AU}$, $e=0.85$; zero ejection velocity is considered. Also a shift of perihelion $\Delta \omega$ (secular change of longitude of perihelion) after 1000 years is given (note that P-R effect with fixed optical properties would yield $\Delta \omega=0$ ). The longitude of perihelion is the angle of perihelion, measured from the ascending node in the direction of the motion of the particle. The last two columns of Table 1 represent values of $\bar{Q}_{\mathrm{pr} \text { aph }}^{\prime}$ at aphelion and $\bar{Q}_{\mathrm{pr} \text { per }}^{\prime}$ at perihelion for the current orbit after 1000 years.

On the basis of the values for the shift of perihelion for particles with radii 2 microns, one would expect that the shift of perihelion for $R=1 \mu \mathrm{m}$ and $\rho=1 \mathrm{~g} / \mathrm{cm}^{3}$ would be greater than the shift of perihelion for $R=1 \mu \mathrm{m}$ and $\rho=2 \mathrm{~g} / \mathrm{cm}^{3}$. However, as the values presented in Table 1 show, this is not true. To correctly understand the result, we have to take into account the real orbital evolution of the particles. It turns out that the particle with $R=1 \mu \mathrm{m}$ and $\rho=1 \mathrm{~g} / \mathrm{cm}^{3}$ moves almost on a circular orbit - eccentricity 0.09 to 0.10 - in a distance $r \approx 4$ AU from the Sun. Since the distance changes only in a very small interval, the changes of efficiency factor for radiation pressure are also small and this is the cause of the small perihelion shift. The other particles presented in Table 1 are characterized by much larger values of eccentricities and perihelion distances are about $0.6 \mathrm{AU}: R=$ $1 \mu \mathrm{m}$ and $\rho=2 \mathrm{~g} / \mathrm{cm}^{3}$ yields $0.54<e<0.74, R=2 \mu \mathrm{m}$ and $\rho=1 \mathrm{~g} / \mathrm{cm}^{3}$ yields $0.57<e<0.76, R=2 \mu \mathrm{m}$, and $\rho=2 \mathrm{~g} / \mathrm{cm}^{3}$ yields $0.70<e<0.81$. Moreover, these particles can move in smaller heliocentric distances, where changes of $\bar{Q}_{\mathrm{pr}}^{\prime}$ are more significant. Thus, the particles exhibit larger changes of $\bar{Q}_{\mathrm{pr}}^{\prime}$ and this also explains the large values of advancement of perihelion. 
Table 2 presents ratios for secular values of semi-major axes and eccentricities after 1000 years, i.e., $a\left[\bar{Q}_{\mathrm{pr} \text { aph }}^{\prime}(0)\right] / a\left[\bar{Q}_{\mathrm{pr}}^{\prime}\right]$, and $e\left[\bar{Q}_{\mathrm{pr} \text { aph }}^{\prime}(0)\right] / e\left[\bar{Q}_{\mathrm{pr}}^{\prime}\right] \cdot \bar{Q}_{\mathrm{pr} \text { aph }}^{\prime}(0)$ is an initial value and $\bar{Q}_{\mathrm{pr}}^{\prime}$ changes in orbital evolution and thus it is a function of the heliocentric distance.

The important data summarized in Tables 1 and 2 lead to the following results. The effect of electromagnetic radiation on spherical micron-sized dust particles yields, in general, a shift of perihelion, while the evolution of the semi-major axis and eccentricity may be calculated within an approximation of constant optical properties (see Eqs. (64)-(65), (102)-(103), with the corresponding initial conditions (58)-(63) in Klačka 2004). The statement about the semi-major axis and eccentricity hold when heliocentric distances are not smaller than about 0.1 AU. Detail numerical solution of Eq. (3) has to be done for orbital evolution if heliocentric distances may be smaller than about $0.1 \mathrm{AU}$.

\section{Discussion}

Tables 1 and 2 present quantities that show the difference between the evolution of orbital elements for fixed values of optical properties and the more realistic values of the optical properties for the Poynting-Robertson effect. The evolution of the semimajor axis and eccentricity may be described for a long time by the approximate secular evolution known since the time of Robertson; the smaller heliocentric distance, the greater changes of $Q_{\mathrm{pr}}^{\prime}$ occurs, see Table 1 and Kocifaj et al. (2006), and the greater differences between real and approximate evolutions exist. The real evolution yields a nonzero shift of perihelion, while fixed optical properties yield zero perihelion motion. However, the value of the perihelion shift presented in Table 1 is also an approximation of the reality. The approximation is in the fact that Eq. (3) is written in the form

$\frac{\mathrm{d} \boldsymbol{v}}{\mathrm{d} t}=-\frac{G M_{\odot}(1-\beta)}{|\boldsymbol{r}|^{3}} \boldsymbol{r}-\beta \frac{G M_{\odot}}{|\boldsymbol{r}|^{2}}\left\{\left(\frac{\boldsymbol{v} \cdot \hat{\boldsymbol{r}}}{c}\right) \hat{\boldsymbol{r}}+\frac{\boldsymbol{v}}{c}\right\}$,

and the osculating elements are calculated for the central acceleration given by the term independent of velocity $\boldsymbol{v}$. In reality, the term $G M_{\odot}(1-\beta)$ is not constant in time, and, the corresponding term does not yield Keplerian orbits. The correct approach is to consider the central acceleration given by gravity only, as it is in Eq. (3) and discussed in detail in Klačka (2004). However, if we want to show that the shift of perihelion already exists when the central acceleration is given by the term $G M_{\odot}(1-\beta)$, then we make an approximation $\beta=\beta_{0}+\beta_{0}^{\prime}\left(r-r_{0}\right)$. We can show that the shift of perihelion exists even for the case when velocity terms in Eq. (4) are neglected. In reality, we have

$\frac{\mathrm{d} \boldsymbol{v}}{\mathrm{d} t}=-\frac{G M_{\odot}\left(1-\beta_{0}\right)}{|\boldsymbol{r}|^{3}} \boldsymbol{r}+\beta_{0}^{\prime} \frac{G M_{\odot}}{|\boldsymbol{r}|^{2}}\left(r-r_{0}\right) \hat{\boldsymbol{r}}$.

The change of longitude of perihelion is given by

$$
\begin{aligned}
\frac{\mathrm{d} \omega_{\beta 0}}{\mathrm{~d} t}= & -\frac{1}{e_{\beta 0}} \sqrt{\frac{p_{\beta 0}}{G M_{\odot}\left(1-\beta_{0}\right)}} \frac{G M_{\odot}}{r^{2}} \\
& \times \beta_{0}^{\prime}\left(r-r_{0}\right) \cos f_{\beta 0},
\end{aligned}
$$

where $f_{\beta 0}$ is a true anomaly (all orbital elements relates to the central acceleration $G M_{\odot}\left(1-\beta_{0}\right)$, also a true anomaly; corresponding quantities are denoted by subscript $\beta 0$, to be consistent with Klačka 2004). Using the fact that the semi-major axis and eccentricity exhibit small changes during one period
Table 2. Ratios of semi-major axes and eccentricities for fixed $\bar{Q}_{\mathrm{pr} \text { aph }}^{\prime}(0)$ and time dependent $\bar{Q}_{\mathrm{pr}}^{\prime}$ values of radiation pressure efficiency factor for carbonaceous spherical dust particles after 1000 years.

\begin{tabular}{ccccc}
\hline \hline$R[\mu \mathrm{m}]$ & 1 & 1 & 2 & 2 \\
$\rho\left[\mathrm{g} / \mathrm{cm}^{3}\right]$ & 1 & 2 & 1 & 2 \\
$\frac{a\left[\bar{Q}_{\mathrm{pr}}^{\prime} \mathrm{aph}(0)\right]}{a\left[\bar{Q}_{\mathrm{pr}}^{\prime}\right]}-1$ & $4.21 \mathrm{E}-3$ & $5.87 \mathrm{E}-3$ & $1.42 \mathrm{E}-3$ & $-2.62 \mathrm{E}-3$ \\
$\frac{e\left[\bar{Q}_{\mathrm{pr} a \mathrm{ph}}(0)\right]}{e\left[\overline{\mathrm{Q}}_{\mathrm{pr}}^{\prime}\right]}-1$ & $6.58 \mathrm{E}-3$ & $1.89 \mathrm{E}-3$ & $7.51 \mathrm{E}-4$ & $-1.08 \mathrm{E}-3$ \\
\hline
\end{tabular}

of revolution around the Sun, we can write for secular change $\left[p_{\beta 0}=a_{\beta 0}\left(1-e_{\beta 0}^{2}\right)\right]$

$$
\begin{aligned}
\left\langle\frac{\mathrm{d} \omega_{\beta 0}}{\mathrm{~d} t}\right\rangle= & -\frac{1}{e_{\beta 0}} \sqrt{\frac{p_{\beta 0}}{G M_{\odot}\left(1-\beta_{0}\right)}} \frac{G M_{\odot} \beta_{0}^{\prime}}{a_{\beta 0}^{2} \sqrt{1-e_{\beta 0}^{2}}} \\
& \times \frac{1}{2 \pi} \int_{0}^{2 \pi}\left(r-r_{0}\right) \cos f_{\beta 0} \mathrm{~d} f_{\beta 0} \\
= & \frac{\beta_{0}^{\prime}}{\sqrt{1-\beta_{0}}} \sqrt{\frac{G M_{\odot}}{a_{\beta 0}}} \\
& \times \frac{\sqrt{1-e_{\beta 0}^{2}}\left(1-\sqrt{1-e_{\beta 0}^{2}}\right)}{e_{\beta 0}^{2}}
\end{aligned}
$$

The obtained result is correct to the first order in $\beta_{0}^{\prime}$ (see Eqs. (113)-(115) in Klačka 2004). Equation (7) shows that $\left\langle\mathrm{d} \omega_{\beta 0} / \mathrm{d} t\right\rangle$ is nonzero and positive if $\beta_{0}^{\prime}$ is positive. The numerical solution of Eq. (3) showed that shifts of perihelion for the case when osculating elements are computed using constant $\beta_{0}$ as a part of central acceleration, and for the case when central acceleration contains $\beta$ instead of $\beta_{0}$ (see Table 1 and Fig. 4 below), are almost equal.

The orientation of the advancement of perihelion depends on the orientation of revolution around the Sun, i.e., whether the orbit is prograde or retrograde. The orientation is given by the value of $X \equiv \operatorname{sign}\left\{-\int_{0}^{2 \pi}\left[\beta(r)-\beta_{0}\right] \cos f_{\beta 0} \mathrm{~d} f_{\beta 0}\right\}$, where $\beta_{0} \equiv$ $\beta\left(r_{0}\right)$ and $r=p_{\beta 0} /\left(1+e_{\beta 0} \cos f_{\beta 0}\right)$. The shift of perihelion is in the orientation of the orbital motion, if $X=+1$. The shift of perihelion is in a reverse orientation if $X=-1$.

As for the change of semi-major axis and eccentricity, Eq. (5) yields

$$
\begin{aligned}
\frac{\mathrm{d} a_{\beta 0}}{\mathrm{~d} t}= & \frac{2 a_{\beta 0} e_{\beta 0}}{1-e_{\beta 0}^{2}} \sqrt{\frac{p_{\beta 0}}{G M_{\odot}\left(1-\beta_{0}\right)}} \frac{G M_{\odot}}{r^{2}} \\
& \times \beta_{0}^{\prime}\left(r-r_{0}\right) \sin f_{\beta 0}, \\
\frac{\mathrm{d} e_{\beta 0}}{\mathrm{~d} t}= & e_{\beta 0} \sqrt{\frac{p_{\beta 0}}{G M_{\odot}\left(1-\beta_{0}\right)}} \frac{G M_{\odot}}{r^{2}} \\
& \times \beta_{0}^{\prime}\left(r-r_{0}\right) \sin f_{\beta 0} .
\end{aligned}
$$

The secular changes are

$\left\langle\frac{\mathrm{d} a_{\beta 0}}{\mathrm{~d} t}\right\rangle=0$
$\left\langle\frac{\mathrm{d} e_{\beta 0}}{\mathrm{~d} t}\right\rangle=0$.

Equation (9) analytically explains the small differences presented in Table 2, calculated for central acceleration containing $G M_{\odot}(1-\beta)$. Table 3 presents the real evolution of the sculating 
Table 3. Ratios of real osculating semi-major axes and eccentricities for fixed $\bar{Q}_{\mathrm{pr} \text { aph }}^{\prime}(0)$ and time dependent $\bar{Q}_{\mathrm{pr}}^{\prime}$ values of radiation pressure efficiency factor for carbonaceous spherical dust particle after 1000 years.

\begin{tabular}{ccccc}
\hline \hline$R[\mu \mathrm{m}]$ & 1 & 1 & 2 & 2 \\
$\rho\left[\mathrm{g} / \mathrm{cm}^{3}\right]$ & 1 & 2 & 1 & 2 \\
$\frac{a\left[\bar{Q}_{\mathrm{pr}}^{\prime} \mathrm{aph}(0)\right]}{a\left[\bar{Q}_{\mathrm{pr}}\right]}-1$ & $7.96 \mathrm{E}-4$ & $-1.64 \mathrm{E}-3$ & $-1.45 \mathrm{E}-3$ & $-3.86 \mathrm{E}-3$ \\
$\frac{e\left[\bar{Q}_{\mathrm{pr}}^{\prime} \mathrm{aph}\right.}{e\left[\bar{Q}_{\mathrm{pr}}^{\prime}\right]}-1$ & $-6.85 \mathrm{E}-3$ & $-1.04 \mathrm{E}-3$ & $-8.68 \mathrm{E}-4$ & $-1.50 \mathrm{E}-3$ \\
\hline
\end{tabular}
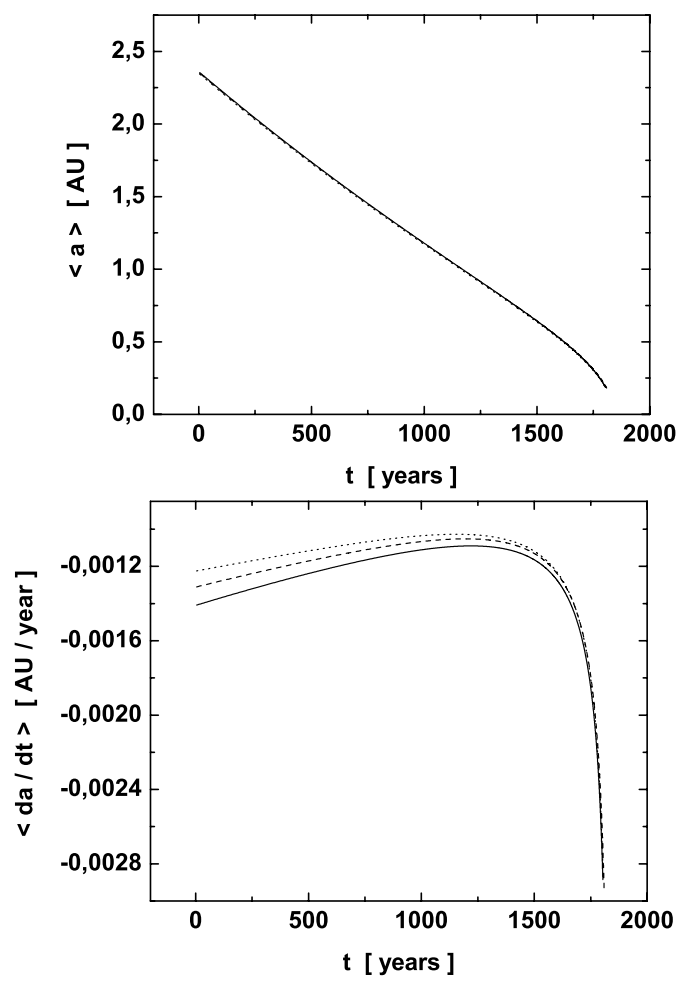

Fig. 2. Secular change of semi-major axis and its time derivative for spherical carbonaceous particle with radius $R=1 \mu \mathrm{m}$ and mass density $\rho=2 \mathrm{~g} / \mathrm{cm}^{3}$.

orbital elements for the P-R effect for the central acceleration containing $G M_{\odot}\left(1-\beta_{0}\right)$. Values presented in Table 3 are also consistent with Eq. (9). (Table 1 holds for both central accelerations: containing $G M_{\odot}(1-\beta)$ and $G M_{\odot}\left(1-\beta_{0}\right)$, too.)

Figures 2-4 present the secular evolution of the semi-major axis, eccentricity, and longitude of perihelion, including the secular evolution of their time derivatives. Three various curves for secular evolution of time derivatives of semi-major axis and eccentricity (for carbonaceous particles with $R=1 \mu \mathrm{m}$, $\varrho=2 \mathrm{~g} / \mathrm{cm}^{3}$ ) correspond to three types of osculating elements: the first type is an element for central acceleration given by $G M_{\odot}(1-\beta(r)) / r^{2}$ (dotted curve), the second type is for the case $G M_{\odot}\left(1-\beta_{\text {aphelion }}\right) / r^{2}$ and $\beta(r)=\beta_{\text {aphelion }}=$ const. (dashed curve), and the third type (solid curve) is for the case $G M_{\odot}\left(1-\beta_{\text {aphelion }}(0)\right) / r^{2}$ and $\beta(r)$ is a function of heliocentric distance. As for the secular evolution of the time derivative of the longitude of perihelion, the case $G M_{\odot}\left(1-\beta_{\text {aphelion }}\right) / r^{2}$ and $\beta(r)=\beta_{\text {aphelion }}=$ constant yields $\langle\mathrm{d} \omega / \mathrm{d} t\rangle=0$. The shift of perihelion does not exist in this case and we may put $\langle\omega\rangle=0$, as it is done in Fig. 4. Figures 2 and 3 show that the secular evolutions of the semi-major axis and eccentricity are practically independent on the type of the "osculating" orbital elements. This is
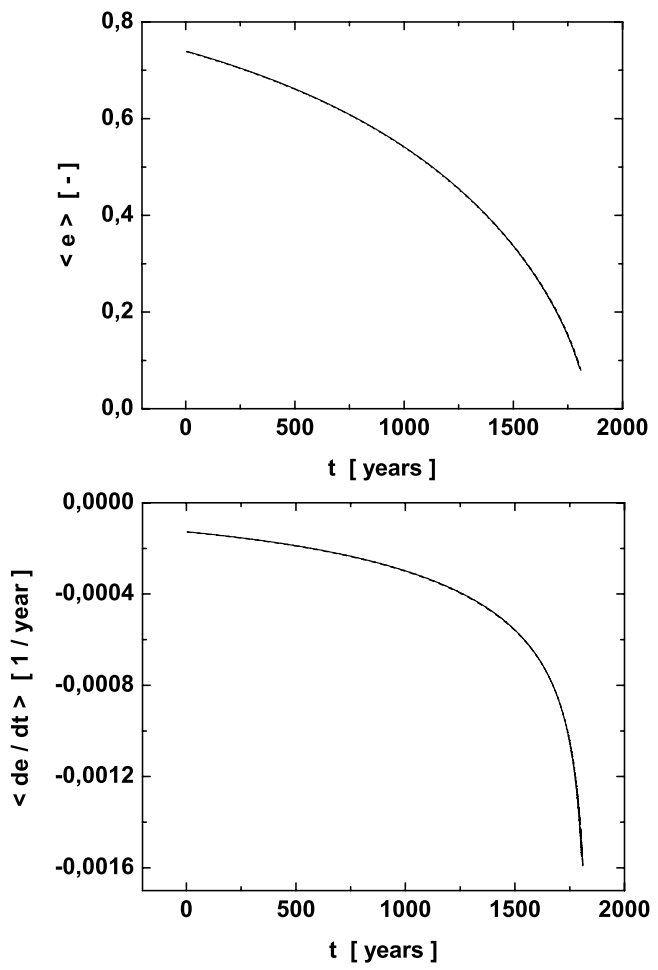

Fig. 3. Secular change of eccentricity and its time derivative for spherical carbonaceous particle with radius $R=1 \mu \mathrm{m}$ and mass density $\rho=2 \mathrm{~g} / \mathrm{cm}^{3}$.

caused by the fact that perturbation term in Eq. (10) (equivalent to Eq. (3))

$$
\begin{aligned}
\frac{\mathrm{d} \boldsymbol{v}}{\mathrm{d} t}= & -\frac{G M_{\odot}\left(1-\beta_{0}\right)}{|\boldsymbol{r}|^{3}} \boldsymbol{r} \\
& +\frac{G M_{\odot}}{|\boldsymbol{r}|^{2}}\left\{\left(\beta-\beta_{0}\right) \hat{\boldsymbol{r}}-\beta\left(\frac{\boldsymbol{v} \cdot \hat{\boldsymbol{r}}}{c} \hat{\boldsymbol{r}}+\frac{\boldsymbol{v}}{c}\right)\right\},
\end{aligned}
$$

$\hat{\boldsymbol{r}} \equiv \boldsymbol{r} /|\boldsymbol{r}|$,

$$
\begin{aligned}
\beta_{0} & \equiv \frac{L_{\odot} \pi R^{2}}{4 \pi G M_{\odot} m_{\mathrm{p}}} \frac{\bar{Q}_{\mathrm{pr} \mathrm{aph}}^{\prime}(0)}{c} \\
& =7.6 \times 10^{-4} \bar{Q}_{\mathrm{pr} \text { aph }}^{\prime}(0) \frac{\pi R^{2}\left[\mathrm{~m}^{2}\right]}{m_{\mathrm{p}}[\mathrm{kg}]},
\end{aligned}
$$

$\beta \equiv \frac{L_{\odot} \pi R^{2}}{4 \pi G M_{\odot} m_{\mathrm{p}}} \frac{\bar{Q}_{\mathrm{pr}}^{\prime}}{c}$

$$
=7.6 \times 10^{-4} \bar{Q}_{\mathrm{pr}}^{\prime} \frac{\pi R^{2}\left[\mathrm{~m}^{2}\right]}{m_{\mathrm{p}}[\mathrm{kg}]},
$$

does not change significantly, unless distances from the Sun are small. This corresponds to the results presented in Eq. (9).

Finally, we want to understand the result presented in Fig. 2, in an analytical way. We will use Eq. (10). We can write

$$
\begin{aligned}
\frac{\mathrm{d} a_{\beta 0}}{\mathrm{~d} t}= & -\beta \frac{G M_{\odot}}{c} \frac{1}{r^{2}} \frac{2 a_{\beta 0}}{1-e_{\beta 0}^{2}} \\
& \times\left\{2\left(e_{\beta 0} \sin f_{\beta 0}\right)^{2}+\left(1+e_{\beta 0} \cos f_{\beta 0}\right)^{2}\right\} \\
& +\left(\beta-\beta_{0}\right) \frac{2 a_{\beta 0} e_{\beta 0}}{1-e_{\beta 0}^{2}} \sqrt{\frac{p_{\beta 0}}{G M_{\odot}\left(1-\beta_{0}\right)}} \\
& \times \frac{G M_{\odot}}{r^{2}} \sin f_{\beta 0} .
\end{aligned}
$$



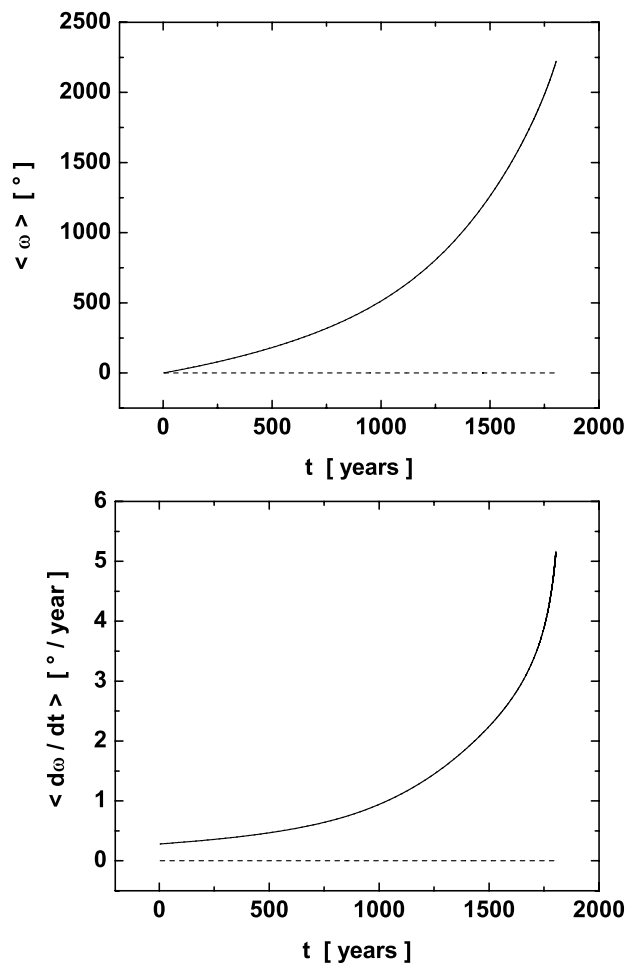

Fig. 4. Secular change of longitude of perihelion and its time derivative for spherical carbonaceous particle with radius $R=1 \mu \mathrm{m}$ and mass density $\rho=2 \mathrm{~g} / \mathrm{cm}^{3}$.

Making the Taylor expansion around the distance $r_{0}$ and taking accuracy to the first power in $r-r_{0}$ into account, we obtain

$$
\begin{aligned}
\frac{\mathrm{d} a_{\beta 0}}{\mathrm{~d} t}= & -\left[\beta_{0}+\beta_{0}^{\prime}\left(r-r_{0}\right)\right] \frac{1}{c} \frac{G M_{\odot}}{r^{2}} \frac{2 a_{\beta 0}}{1-e_{\beta 0}^{2}} \\
& \times\left\{2\left(e_{\beta 0} \sin f_{\beta 0}\right)^{2}+\left(1+e_{\beta 0} \cos f_{\beta 0}\right)^{2}\right\} \\
& +\beta_{0}^{\prime}\left(r-r_{0}\right) \sqrt{\frac{p_{\beta 0}}{G M_{\odot}\left(1-\beta_{0}\right)}} \\
& \times \frac{G M_{\odot}}{r^{2}} \frac{2 a_{\beta 0} e_{\beta 0}}{1-e_{\beta 0}^{2}} \sin f_{\beta 0} .
\end{aligned}
$$

Similarily, the change of longitude of perihelion is

$$
\begin{aligned}
\frac{\mathrm{d} \omega_{\beta 0}}{\mathrm{~d} t}= & -\frac{1}{e_{\beta 0}} \sqrt{\frac{p_{\beta 0}}{G M_{\odot}\left(1-\beta_{0}\right)}} \frac{G M_{\odot}}{r^{2}} \\
& \times\left\{\beta_{0}^{\prime}\left(r-r_{0}\right) \cos f_{\beta 0}+\left[\beta_{0}+\beta_{0}^{\prime}\left(r-r_{0}\right)\right]\right. \\
& \times \frac{\sqrt{G M_{\odot}\left(1-\beta_{0}\right) / p_{\beta 0} 0}}{c} \\
& \left.\times\left(2-e_{\beta 0} \cos f_{\beta 0}\right) \sin f_{\beta 0}\right\} .
\end{aligned}
$$

Making standard averaging

$$
\left\langle\frac{\mathrm{d} a_{\beta 0}}{\mathrm{~d} t}\right\rangle=\frac{1}{a_{\beta 0}^{2} \sqrt{1-e_{\beta 0}^{2}}} \frac{1}{2 \pi} \int_{0}^{2 \pi} r^{2} \frac{\mathrm{d} a_{\beta 0}}{\mathrm{~d} t} \mathrm{~d} f_{\beta 0} .
$$

Equation (12) yields

$$
\begin{aligned}
\left\langle\frac{\mathrm{d} a_{\beta 0}}{\mathrm{~d} t}\right\rangle= & -\beta_{0} \frac{G M_{\odot}}{c} \frac{2+3 e_{\beta 0}^{2}}{a_{\beta 0}\left(1-e_{\beta 0}^{2}\right)^{3 / 2}} \\
& \times\left\{1-\frac{\beta_{0}^{\prime}}{\beta_{0}}\left[r_{0}-2 a_{\beta 0}\left(1-e_{\beta 0}^{2}\right)\right.\right. \\
& \left.\left.\times \frac{3-2 \sqrt{1-e_{\beta 0}^{2}}}{2+3 e_{\beta 0}^{2}}\right]\right\} .
\end{aligned}
$$

If we take $r_{0}$ as an aphelion distance and if we take into account that $\beta_{0}^{\prime}=2.689 \times 10^{-3} \mathrm{AU}^{-1}$ (positive value, see also Fig. 1), then Eq. (12) already immediately states that $\left\langle\mathrm{d} a_{\beta 0} / \mathrm{d} t\right\rangle>$ $\left\langle\mathrm{d} a_{\beta 0} / \mathrm{d} t\right\rangle\left(\beta_{0}^{\prime} \equiv 0\right)$; precisely, the values $\beta_{0}=0.4156, a_{\beta 0}$ in $=$ $2.3546 \mathrm{AU}, e_{\beta} 0$ in $=0.7391$ yield, on the basis of Eq. (15), $\left\langle\mathrm{d} a_{\beta 0} / \mathrm{d} t\right\rangle=-1.288 \times 10^{-3} \mathrm{AU} / \mathrm{yr}$, while $\left\langle\mathrm{d} a_{\beta 0} / \mathrm{d} t\right\rangle\left(\beta_{0}^{\prime} \equiv 0\right)=$ $-1.314 \times 10^{-3} \mathrm{AU} / \mathrm{yr}$. This is not consistent with Fig. 2, according to which $\left\langle\mathrm{d} a_{\beta 0} / \mathrm{d} t\right\rangle\left\langle\left\langle\mathrm{d} a_{\beta 0} / \mathrm{d} t\right\rangle\left(\beta_{0}^{\prime} \equiv 0\right)\right.$ (compare solid and dashed curves for time $t=0$ ).

We can improve the result represented by Eq. (15). We will use a more precise type of averaging, instead of Eq. (14):

$$
\begin{aligned}
& \left\langle\frac{\mathrm{d} a_{\beta 0}}{\mathrm{~d} t}\right\rangle=\frac{\int_{0}^{2 \pi} A \frac{\mathrm{d} a_{\beta 0}}{\mathrm{~d} t} \mathrm{~d} f_{\beta 0}}{\int_{0}^{2 \pi} A \mathrm{~d} f_{\beta 0}}, \\
& A \equiv\left(\frac{\sqrt{G M_{\odot}\left(1-\beta_{0}\right) p_{\beta 0}}}{r^{2}}-\frac{\mathrm{d} \omega_{\beta 0}}{\mathrm{~d} t}\right)^{-1} .
\end{aligned}
$$

Equations (12), (13), and (16) yield

$$
\begin{aligned}
\left\langle\frac{\mathrm{d} a_{\beta 0}}{\mathrm{~d} t}\right\rangle= & -\beta_{0} \frac{G M_{\odot}}{c} \frac{2+3 e_{\beta 0}^{2}}{a_{\beta 0}\left(1-e_{\beta 0}^{2}\right)^{3 / 2}} \\
& \times\left\{1-\frac{\beta_{0}^{\prime}}{\beta_{0}}\left[r_{0}-2 p_{\beta 0} \frac{3-2 \sqrt{1-e_{\beta 0}^{2}}}{2+3 e_{\beta 0}^{2}}\right]\right. \\
& +\frac{\beta_{0}^{\prime}}{1-\beta_{0}}\left[2 p_{\beta 0} \frac{5\left(1-\sqrt{1-e_{\beta 0}^{2}}\right)-2 e_{\beta 0}^{2}}{\left(2+3 e_{\beta 0}^{2}\right) e_{\beta 0}^{2}}\right. \\
& \left.\left.+r_{0}-\frac{3}{2} a_{\beta 0}\right]\right\} .
\end{aligned}
$$

Equation (17) yields the value $\left\langle\mathrm{d} a_{\beta 0} / \mathrm{d} t\right\rangle=-1.295 \times$ $10^{-3} \mathrm{AU} / \mathrm{yr}$. This value is smaller than the value obtained on the basis of Eq. (15), but it is greater than the value for the case $\beta_{0}^{\prime} \equiv$ 0 . The last statement is not consistent with our detailed numerical calculations, as it is presented in Fig. 2. It seems that the difference between the detailed numerical calculations and our analytical approach is caused by real changes of $\bar{Q}_{\mathrm{pr}}^{\prime}$ as a function of heliocentric distance and any simple approximation of this function does not reproduce the real behavior of $\left\langle\mathrm{d} a_{\beta 0} / \mathrm{d} t\right\rangle$. If we would use the Taylor expansion up to the third power in $r-r_{0}$, then $\left|\beta_{0}^{\prime \prime}\right|$ and $\left|\beta_{0}^{\prime \prime \prime}\right|$ are comparable to $\left|\beta_{0}^{\prime}\right|:\left|\beta_{0}^{\prime \prime \prime}\right| \approx\left|\beta_{0}^{\prime \prime}\right| / 2 \approx\left|\beta_{0}^{\prime}\right| / 8$.

Now, we want to understand the other property of the function presented in Fig. 2: the initial increase of $\left\langle\mathrm{d} a_{\beta 0} / \mathrm{d} t\right\rangle$ turns out to be a decrease at final stages of the orbital evolution. We will use $\beta_{0}^{\prime} \equiv 0$ in our considerations. Using the secular changes for the semi-major axis and eccentricity for the constant optical 
properties for the P-R effect (Robertson 1937; Wyatt \& Whipple 1950; Klačka 2004)

$\left\langle\frac{\mathrm{d} a_{\beta 0}}{\mathrm{~d} t}\right\rangle=-\beta_{0} \frac{G M_{\odot}}{c} \frac{2+3 e_{\beta 0}^{2}}{a_{\beta 0}\left(1-e_{\beta 0}^{2}\right)^{3 / 2}}$,

$\left\langle\frac{\mathrm{d} e_{\beta 0}}{\mathrm{~d} t}\right\rangle=-\frac{5}{2} \beta_{0} \frac{G M_{\odot}}{c} \frac{e_{\beta 0}}{a_{\beta 0}^{2}\left(1-e_{\beta 0}^{2}\right)^{1 / 2}}$,

we can find a maximum of $\left\langle\mathrm{d} a_{\beta 0} / \mathrm{d} t\right\rangle$. We want to show that it really corresponds to the value that can be seen in Fig. 2 on a dashed line. The derivative of the secular change of the semimajor axis is

$\frac{\mathrm{d}}{\mathrm{d} t}\left\langle\frac{\mathrm{d} a_{\beta 0}}{\mathrm{~d} t}\right\rangle=-\frac{1}{2} \beta_{0}^{2} \frac{G^{2} M_{\odot}^{2}}{c^{2}} \frac{8-36 e_{\beta 0}^{2}+3 e_{\beta 0}^{4}}{a_{\beta 0}^{3}\left(1-e_{\beta 0}^{2}\right)^{3}}$.

The maximum of $\left\langle\mathrm{d} a_{\beta 0} / \mathrm{d} t\right\rangle$ yields $8-36 e_{\beta 0}^{2}+3 e_{\beta 0}^{4}=0$. The obtained quadratic equation yields only one solution for $e_{\beta 0} \in$ $\left(0, e_{\beta} 0\right.$ in $)$ :

$e_{\beta 0 \mathrm{~m}}=\sqrt{6-10 / \sqrt{3}}=0.4759$.

Using the relationship between the semi-major axis and eccentricity

$a_{\beta 0}=a_{\beta 0 \text { in }} \frac{1-e_{\beta 0 \text { in }}^{2}}{1-e_{\beta 0}^{2}}\left(\frac{e_{\beta 0}}{e_{\beta 0 \text { in }}}\right)^{4 / 5}$,

and inserting Eqs. (21) and (22) into Eq. (18), we get

$$
\begin{aligned}
\left\langle\frac{\mathrm{d} a_{\beta 0}}{\mathrm{~d} t}\right\rangle_{\max }= & -\beta_{0} \frac{G M_{\odot}}{c} \frac{1}{a_{\beta 0 \text { in }}\left(1-e_{\beta 0 \text { in }}^{2}\right)} \\
& \times\left(\frac{e_{\beta 0 \text { in }}}{e_{\beta 0 \mathrm{~m}}}\right)^{4 / 5} \frac{2+3 e_{\beta 0 \mathrm{~m}}^{2}}{\left(1-e_{\beta 0 \mathrm{~m}}^{2}\right)^{1 / 2}} .
\end{aligned}
$$

The case in Fig. 2 is characterized by $\beta=0.4156, a_{\beta} 0$ in $=$ $2.3546 \mathrm{AU}$, and $e_{\beta} 0$ in $=0.7391$ (mean values of the first revolution are: $\left.\left\langle a_{\beta 0}\right\rangle=2.3484 \mathrm{AU},\left\langle e_{\beta} 0\right\rangle=0.7385\right)$. Equation (23) gives $\left\langle\mathrm{d} a_{\beta 0} / \mathrm{d} t\right\rangle_{\max }=-1.0515 \times 10^{-3} \mathrm{AU} / \mathrm{yr}$. All calculations presented in Eqs. (18)-(23), including the last numerical value, are consistent with Fig. 2. We can derive a similar equation for the eccentricity

$\frac{\mathrm{d}}{\mathrm{d} t}\left\langle\frac{\mathrm{d} e_{\beta 0}}{\mathrm{~d} t}\right\rangle=-\frac{15}{4} \beta_{0}^{2} \frac{G^{2} M_{\odot}^{2}}{c^{2}} \frac{1+4 e_{\beta 0}^{2}}{a_{\beta 0}^{4}\left(1-e_{\beta 0}^{2}\right)^{2}}<0$.

This is in accordance with our simulations that $\left\langle\mathrm{d} e_{\beta 0} / \mathrm{d} t\right\rangle$ is a monotonic and a decreasing function of time (see Fig. 3).

Finally, we want to find the most simple analytic form of the differential equation for secular evolution of the shift of pericenter. If the change of optical properties can be approximated as $\beta=\beta_{0}+\beta_{0}^{\prime}\left(r-r_{0}\right)$, then Eqs. (7), (18), (19), and (22) yield

$$
\begin{aligned}
\left\langle\frac{\mathrm{d} \omega_{\beta 0}}{\mathrm{~d} t}\right\rangle= & \frac{\beta_{0}^{\prime}}{\sqrt{1-\beta_{0}}} \sqrt{\frac{G M_{\star}}{p_{\beta 0 \text { in }} / e_{\beta 0 \text { in }}^{4 / 5}}} \\
& \times \frac{\left(1-e_{\beta 0}^{2}\right)\left(1-\sqrt{1-e_{\beta 0}^{2}}\right)}{e_{\beta 0}^{12 / 5}},
\end{aligned}
$$
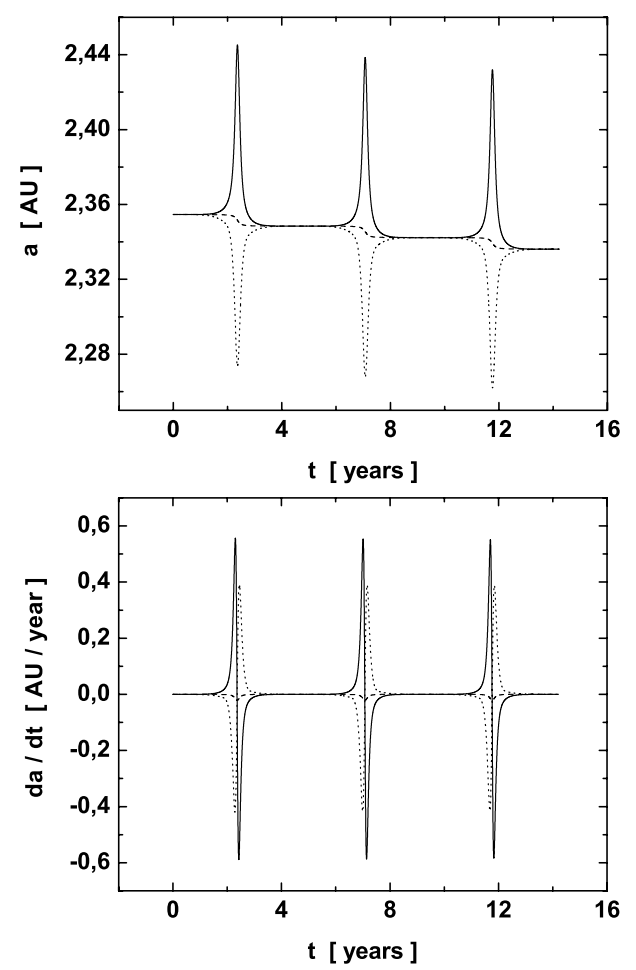

Fig. 5. Evolution of the semi-major axis and its time derivative during the first three revolutions for spherical carbonaceous particle with radius $R=1 \mu \mathrm{m}$ and mass density $\rho=2 \mathrm{~g} / \mathrm{cm}^{3}$.

$$
\begin{aligned}
& \left\langle\frac{\mathrm{d} e_{\beta 0}}{\mathrm{~d} t}\right\rangle=-\frac{5}{2} \beta_{0} \frac{G M_{\star}}{c}\left(\frac{p_{\beta 0 \text { in }}}{e_{\beta 0 \text { in }}^{4 / 5}}\right)^{-2} \frac{\left(1-e_{\beta 0}^{2}\right)^{3 / 2}}{e_{\beta 0}^{3 / 5}}, \\
& a_{\beta 0}=\frac{p_{\beta 0 \text { in }}}{e_{\beta 0 \text { in }}^{4 / 5}} \frac{e_{\beta 0}^{4 / 5}}{1-e_{\beta 0}^{2}}
\end{aligned}
$$

where $M_{\star}$ is mass of the central star. Equation (25) qualitatively describes orbital evolution of the spherical dust grain under the action of electromagnetic radiation generated by the central star. Equation (25) may be rewritten in the following form:

$$
\begin{aligned}
& \omega_{\beta 0}=\omega_{\beta 0 \text { in }}-\frac{2}{5} \frac{\beta_{0}^{\prime}}{\beta_{0} \sqrt{1-\beta_{0}}} \frac{1}{\sqrt{G M_{\star}}} \\
& \times\left(\frac{p_{\beta 0 \text { in }}}{e_{\beta 0 \text { in }}^{4 / 5}}\right)^{3 / 2} \quad \int_{e_{\beta 0 \text { in }}}^{e_{\beta 0}} \frac{1-\sqrt{1-x^{2}}}{x^{9 / 5} \sqrt{1-x^{2}}} \mathrm{~d} x, \\
& \left\langle\frac{\mathrm{d} e_{\beta 0}}{\mathrm{~d} t}\right\rangle=-\frac{5}{2} \beta_{0} \frac{G M_{\star}}{c}\left(\frac{p_{\beta 0 \text { in }}}{e_{\beta 0 \text { in }}^{4 / 5}}\right)^{-2} \frac{\left(1-e_{\beta 0}^{2}\right)^{3 / 2}}{e_{\beta 0}^{3 / 5}}, \\
& a_{\beta 0}=\frac{p_{\beta 0 \text { in }}}{e_{\beta 0 \text { in }}^{4 / 5}} \frac{e_{\beta 0}^{4 / 5}}{1-e_{\beta 0}^{2}} \text {. }
\end{aligned}
$$

Finally, Figs. 5-7 document interesting behavior of the semimajor axis, eccentricity, and longitude of perihelion (and their derivatives) of the carbonaceous particle during the first three revolutions around the Sun after the particle's ejection from the parent body. The case with central acceleration containing $G M_{\odot}\left(1-\beta_{0}\right)$ and $\beta=\beta(r)$ is depicted by a solid line. The case when the central acceleration contains $G M_{\odot}\left(1-\beta_{0}\right)$ and $\beta(r) \equiv \beta_{0}$ is the only case when the evolution of the semi-major axis is a monotonous function of time (a decreasing function, 

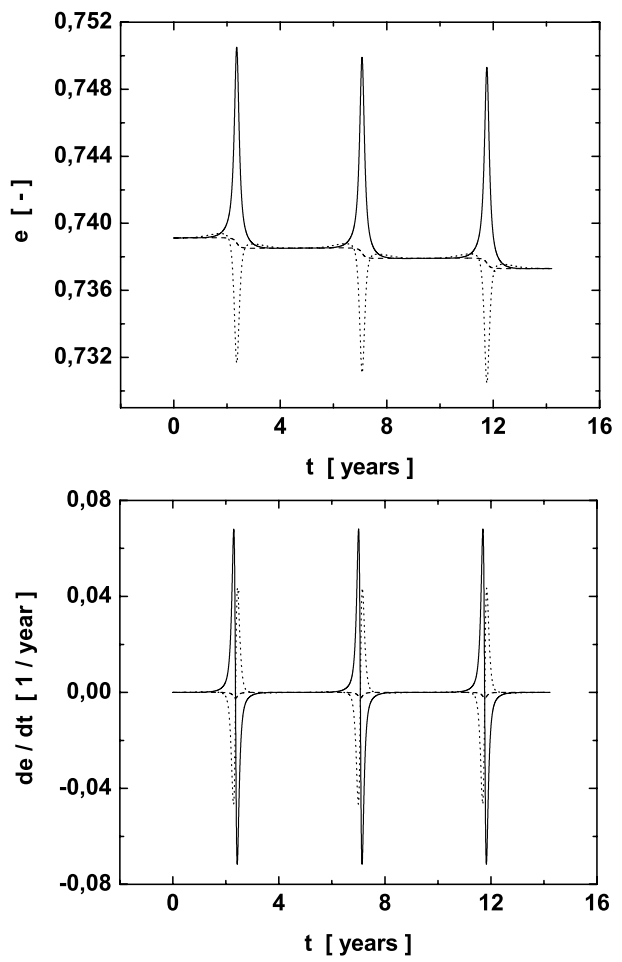

Fig. 6. Evolution of the eccentricity and its time derivative during the first three revolutions for spherical carbonaceous particle with radius $R=1 \mu \mathrm{m}$ and mass density $\rho=2 \mathrm{~g} / \mathrm{cm}^{3}$.

$\left.\mathrm{d} a_{\beta 0} / \mathrm{d} t<0\right)$ as it is easily seen from Eq. (11). The case with central acceleration containing $G M_{\odot}[1-\beta(r)]$ is depicted by dotted line; this case does not correspond to orbital elements in the sense of Keplerian orbits. The evolution of the time derivative of the semi-major axis, for the case when central acceleration contains $G M_{\odot}\left(1-\beta_{0}\right)$ and $\beta=\beta(r)$, corresponds to Eq. (11).

The shifts of perihelia for particles of radii $1 \mu \mathrm{m}$ are, approximately, 1-2 orders of magnitude larger than for particles having sizes $10-50 \mu \mathrm{m}$. As an example, the shifts of compact homogeneous spheres are presented in Table 4 for particle radii $5 \mu \mathrm{m}$ and $25 \mu \mathrm{m}$. A simple consequence of Table 4 is that the shift of perihelion is more important for particles of smaller mass densities (greater $\beta$ ). Moreover, $\beta_{0}^{\prime}$ is greater for small heliocentric distances and, thus, orbits with smaller heliocentric distances exhibit greater shifts of perihelia. Porous dust grains exhibit optical properties that may significantly differ from compact grains (e.g., Mukai et al. 1992; Saija et al. 2005; Vilaplana et al. 2006). Various calculations yield that fluffy aggregates do not show a strong decrease of $\beta$ with increasing particle size (Mukai et al. 1982). Thus, shifts of perihelia of porous spherical particles may be important even for diameters of several tens of micrometers.

An interesting result is obtained from comparison between behavior of spherical and nonspherical particles under the action of electromagnetic radiation of a central star. At first, we can immediately state that the shift of pericenter exists for arbitrarily shaped dust particles, already for the equation of motion considered only in the lowest order in $v / c$ (see Eqs. (41), (42) in Klačka 2004). While for nonspherical dust grains the shift of pericenter also exists for the approximation of the fixed optical properties, the advancement of pericenter of spherical particles exists only when temperature dependent optical properties are considered. As the results of our paper and the paper by Klačka et al. (2006) show, spherical grains may exhibit larger motions of pericenter than nonspherical grains; the result
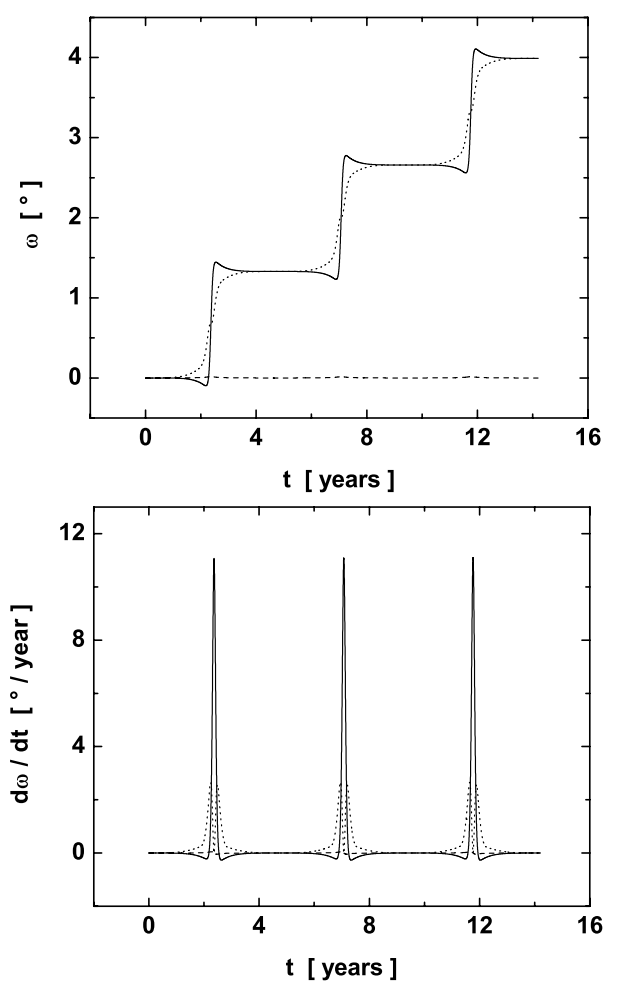

Fig. 7. Evolution of the longitude of perihelion and its time derivative during the first three revolutions for spherical carbonaceous particle with radius $R=1 \mu \mathrm{m}$ and mass density $\rho=2 \mathrm{~g} / \mathrm{cm}^{3}$.

Table 4. Shifts of perihelia for particles of radii $5 \mu \mathrm{m}$ and $25 \mu \mathrm{m}$. The values of $\Delta \omega_{1}$ correspond to the particle mass density $\rho=1 \mathrm{~g} \mathrm{~cm}^{-3}$, while $\Delta \omega_{2}$ was obtained for $\rho=2 \mathrm{~g} \mathrm{~cm}^{-3}$. The particle hits the Sun after: $(*) 3.4 \times 10^{3} \mathrm{yr},(* *) 6.1 \times 10^{3} \mathrm{yr},(* * *) 1.6 \times 10^{4} \mathrm{yr}$.

\begin{tabular}{ccccc}
\hline \hline$t\left[10^{3} \mathrm{yr}\right]$ & \multicolumn{2}{c}{$R=5 \mu \mathrm{m}$} & \multicolumn{2}{c}{$R=25 \mu \mathrm{m}$} \\
& $\Delta \omega_{1}\left[^{\circ}\right]$ & $\Delta \omega_{2}\left[^{\circ}\right]$ & $\Delta \omega_{1}\left[{ }^{\circ}\right]$ & $\Delta \omega_{2}\left[^{\circ}\right]$ \\
\hline 1 & 58.7 & 25.3 & 6.6 & 3.2 \\
2 & 197.6 & 62.4 & 14.1 & 6.6 \\
3 & 585.6 & 122.8 & 22.7 & 10.2 \\
4 & $(*)$ & 229.9 & 32.7 & 14.1 \\
5 & & 416.5 & 44.5 & 18.3 \\
6 & & 755.4 & 58.3 & 22.7 \\
10 & & $(* *)$ & 147.1 & 44.7 \\
15 & & & 407.5 & 85.2 \\
20 & & & $(* * *)$ & 149.8 \\
\hline
\end{tabular}

strongly depends on optical properties of the particles - aspect ratio, exact shape and porosity of the particle, (complex) refractive index and its temperature dependence, and dimension of the particle - and also on the grains' distances from the central star and the density function of the stellar radiance as a function of a wavelength. On the basis of the paper one could state that the shift of pericenter of a nonspherical dust grain is larger than the shift found by Klačka et al. (2006). However, the numerical simulations on nonspherical particles with temperature dependent dielectric functions are extremely complex. The reason is that irregularly shaped particles have radiation pressure force components that are perpendicular to the direction of irradiation, which is due to the asymmetrical light scattering. Although the transverse force components are small in comparison with the radial radiation force (Il'in \& Voshchinnikov 1998; Krauss \& Wurm 2004, 2005), they may be even more important than $\boldsymbol{v} / c$ 
and thus can markedly influence the dynamical evolution of the particles (Klačka \& Kocifaj 2001). As for numerical runs, there are no problems with spherical particle: its optical properties do not depend on the orientation of particle rotation axis and thus it is sufficient to characterize the radiation pressure by a single value. The temperature of the particle changes with heliocentric distance. It results in the fact that the radiation pressure on a spherical particle is a function of the distance from the Sun. Unfortunately, the radiation pressure on the irregularly shaped particle depends on the actual orientation of the particle in space. An orientation can be characterized by three Euler angles $\vartheta, B$, and $\varphi$. To keep the numerical simulations accurate, one must recompute radial and transversal radiation pressure factors at satisfactory dense lattice $[\vartheta, B, \varphi]$ and for all selected heliocentric distances. This requires an enormous numerical effort to get the corresponding optical properties (see Draine \& Flatau 2004) and there are also problems with great memory consumption. Contrary to the radial component, the transversal components of radiation pressure vary rapidly with both particle orientation and particle temperature, and therefore an interpolation on the grid $[\vartheta, B, \varphi]$ needs robust data sets to obtain reliable conclusions.

Acknowledgements. This work was partially supported by the Scientific Grant Agency VEGA (grant No. 1/3074/06), Grant Agency APVV (grant APVV-0426-06), and by the scientific project of the Comenius University UK/416/2006.

\section{References}

Bohren, C. F., \& Huffman, D. R. 1983, Absorption and Scattering of Light by Small Particles (New York: John Wiley \& Sons, Inc.)
Draine, B. T., \& Flatau, P. J. 2004, User Guide for the Discrete Dipole Approximation Code DDSCAT.6.1, Freeware,

http://arxiv.org/abs/astro-ph/0409262

Draine, B. T., \& Lee, H. M. 1984, ApJ, 285, 89

Il'in, V. B., \& Voshchinnikov, N. V. 1998, A\&AS, 128, 187

Jäger, C., Il'in, V. B., Henning, Th., et al. 2003, J. Quant. Spectrosc. Rad. Transf., 79,765

Klačka, J. 1992, Earth, Moon, and Planets, 59, 41

Klačka, J. 1993, Earth, Moon, Planets, 62, 239

Klačka, J. 2004, Cel. Mech. Dynam. Astron., 89, 1

Klačka, J., \& Kocifaj, M. 2001, J. Quant. Spectrosc. Rad. Transf., 70, 595

Klačka, J., Kocifaj, M., \& Kundracík, F. 2006, Planet. Space. Sci., 54, 379

Kocifaj, M., Klačka, J., \& Horvath, H. 2006, MNRAS, 370, 1876

Krauss, O., \& Wurm, G. 2004, J. Quant. Spectrosc. Rad. Transf., 89, 179

Krauss, O., \& Wurm, G. 2005, Experimental determination of the radiation pressure forces on an individual dust particle, in Dust in Planetary Systems, Proc. of the conference held Sept. 26-28, 2005, in Kana'i, Hawai, LPI contribution No. 1280, 96

Lamy, P. L. 1974, A\&A, 35, 197

Leinert, Ch., \& Grün, E. 1990, Interplanetary dust, in Physics of the Inner Heliosphere I, ed. R. Schwen, \& E. Marsch (Berlin: Springer-Verlag), 207 Lyttleton, R. A. 1976, Ap\&SS, 44, 119

Mie, G. 1908, Ann. Phys., 25, 377

Mishchenko, M. I. 1991, Ap\&SS, 180, 163

Mukai, T., Ishimoto, H., Kozasa, T., Blum, J., \& Greenberg, J. M. 1992, A\&A, 262,315

Quinn, T. 2005, Planet formation, in Chaos and Stability in Planetary Systems, ed. R. Dvorak, F. Freistetter, \& J. Kurths (Berlin: Springer-Verlag), 187

Robertson, H. P. 1937, MNRAS, 97, 423

Saija, R., Iatì, M. A., Giusto, A., Denti, P., \& Borghese, F. 2005, J. Quant. Spectrosc. Rad. Transf., 94, 163

Shulman, L. M. 1987, Cometary nuclei (Moscow: Nauka, in Russian)

Vilaplana, R., Moreno, F., \& Molina, A. 2006, J. Quant. Spectrosc. Rad. Transf., 100,415

Wyatt, S. P., \& Whipple, F. L. 1950, ApJ, 111, 134 